\title{
TRANSPALATAL DISTRACTION OSTEOGENESIS VERSUS HYRAX APPLIANCE FOR CORRECTION OF UNILATERAL MAXILLARY HYPOPLASIA IN CLEFT PALATE PATIENTS
}

\author{
Ibrahim Mohamed Nowair*, Mohamed Kamal Eid** and Ahamed Mostafa Elsharif**
}

\begin{abstract}
Background: Collapsed maxilla in unilateral cleft palate patients results in unilateral crossbite, tapered maxilla and crowding of the maxillary teeth. Distraction osteogenesis (DO) of collapsed maxilla in cleft palate patients has become the contemporary surgical management of many secondary cleft deformities. Purpose: the aim was to compare between the efficiency of using Transpalatal Distraction Osteogenesis (TPDO) and Hyrax appliance for three dimension correction of maxillary hypoplasia in unilateral cleft palate patients both clinically, radiographicaly and study cast analysis. Patients and methods: This study was comprised eight patients group1 (TPDO); (3) males and (5) females and eight patients group2 (Hyrax); (2) males and (6) females with unilateral maxillary hypoplasia after repair of unilateral cleft lip and palate. The age ranged from 13 to 27 years with a mean of 16.88 years. Patients were assessed clinically regarding: photographic, healing of the soft tissue, pain, and device loosening. Radiographically regarding: PA cephalometric, lateral cephalometric views and C.T scan. Study cast regarding: inter canine, inter premolar, and inter molar distance, palatal depth and width. Finally, the data were collected, tabulated and statistically analyzed. Results: The result of this study shows significant difference in inter canine, inter premolar and inter molar distances, palatal width and depth between the pre and post distraction in group (1) than in group (2).
\end{abstract}

Keywords: Unilateral cleft palate patients, transverse maxillary hypoplasia, transpalatal distraction osteogenesis and hyrax appliance.

\section{INTRODUCTION}

Patients with repaired unilateral cleft lip and palate present with large osseous defects of the alveolus and mid facial hypoplasia. Midface deficiency of these patients is normally seen in all three planes that are vertical, sagittal and transverse. In addition, all patients present with scarring, residual fistulae and dental anomalies. All these complications present numerous challenges to the reconstructive surgeon ${ }^{(1)}$. Traditional orthognathic surgery and craniofacial reconstruction have gained

\footnotetext{
* Associate Professor Oral and Maxillofacial Surgery, Faculty of Dentistry, Tanta University, Egypt

** Lecturer Oral and Maxillofacial Surgery, Faculty of Dentistry, Tanta University, Egypt
} 
generally acceptance but with severe limitations have been seen. One of the major limitations is the inability of the soft tissue to stretch leading to relapse $^{(2)}$. Distraction osteogenesis (DO) a recently developed technique seems to solve most of these problems, as it is a well-known fact that bone is a regenerative organ and has an inherent capacity to restore its form and function. DO utilize this capacity of bone as its basic principle along with the soft tissue improvement ${ }^{(3)}$.

Traditionally, a tooth - borne orthodontic appliance called a Hyrax expander is placed preoperatively to expand the maxilla. Dental anchorage give rise to several complications, including damage to the teeth, possible loss of anchorage, periodontal membrane compression and buccal root resorption, cortical fenestration and anchorage - tooth tipping. Advantage of the Hyrax expander include its ability to be placed and removed in the orthodontic outpatient clinic without local anesthesia ${ }^{(4)}$.

Transpalatal distraction osteogenesis (TPDO) is a new method for treating transversal maxillary deficiency using the DO procedure, which has proven very valuable in other surgical fields. TPD device is a bone-born appliance that directs the forces mainly to the palatal shelves close to the center of resistance of the maxillary bone without tooth movement; it also leaves all of the crowns clear for orthodontic access ${ }^{(5)}$. Additionally, most of the maxillary expansion is orthopaedic ${ }^{(6,7)}$.

Transverse maxillary expansion with a bone-born TPD has been used on a limited number of patients with favorable results in congenital and acquired transverse maxillary deficiency ${ }^{(4,8)}$. So, this study was designed for more detailed assessment of the efficacy of using TPDO and Hyrax appliance for 3D correction of maxillary hypoplasia in unilateral cleft palate patients both clinically and radiographically.

\section{PATIENTS AND METHODS}

\section{Sixteen patients randomly divided into two equal groups:}

- Group I (TPDO); (3) males and (5) females

- Group II (Hyrax); (2) males and (6) females

All patients suffered from presence of transverse maxillary deficiency as a result of previously treated unilateral cleft palate. Patients were selected from the Out-Patient Clinic of Oral and Maxillofacial Surgery Department, Faculty of Dentistry, Tanta University. Their age ranged from 13 to 27 years with a mean of (16.88 years).

Before any procedure the patient's informed consent was obtained. The proposed operation or investigations were explained in simple language which could be understood by the patients or their relatives. The more common complications were mentioned without causing undue distress.

Inclusion criteria comprised those patients whose age was not less than thirteen years, alveolar clefts were either previously repaired or inadequately repaired or even unrepaired, and patients with previously repaired unilateral cleft palate with unilateral cross bite more than $5 \mathrm{~mm}$ which failed to be orthodontically treated.

Exclusion Criteria comprised syndromic cases and those with diseases which interfere with bone healing e.g. vitamin D deficiency, connective tissue diseases, metabolic disorders, calcium deficiency, immune-compromised, uncontrolled diabetes, steroid therapy, and irradiation.

\section{Pre-operative evaluation:}

\section{A) Clinical evaluation}

Case history of the patient was taken according to a prepared sheet for determination of age, sex, past medical history to exclude any patient having a systemic disease that might interfere with the results of the study, past dental history especially 
the number and the types of previous operations made for cleft lip, palate and alveolus repair, and the patient's chief complaint or the parent's reason for seeking the surgery. Teeth related to the cleft site, if hopeless for treatment or supernumerary teeth must be extracted at least one month before surgery to secure soft tissue healing before osteotomy.

\section{Extra oral photography}

- Frontal view was acquired to show the asymmetry of the middle face.

- Profile view was acquired to show the sagittal deficiency of the patients.

- Three quarters view of the affected side was acquired to show changes of the nasolabial valley, lip and cheek and monitor the progress of the patients postoperatively.

\section{Intraoral photography}

- Frontal closed view was acquired to show the cross bite in relation to the normal side.

- Frontal open view was acquired to show the collapsed maxilla on the cleft side, the presence of residual oro-nasal fistula and the malposed teeth or supernumerary teeth at cleft site.

- Posterior right and left views were used to detect the side of the cross bite.

\section{B) Radiographic evaluation}

- Periapical x-ray to identify the apices of the teeth at the osteotomy site according to Anttila et al. ${ }^{(9)}$.

- Upper occlusal projection was acquired to identifythe palatally malposed and impacted teeth according to Doruk et al. ${ }^{(10)}$.

- Panoramic radiographic acquisition was used to determine any pathology in both jaws, impacted teeth related to the alveolar cleft and osteotomy site and the proposed osteotomy site where it was above the apices of the roots by at least 3-5 mm according to kuodstal et al. ${ }^{(11)}$.

- Postero-anterior cephalogram was used for evaluating the transverse deficiency preoperatively and monitoring the progress of the patients postoperatively as described in (Figures 1: A, B).

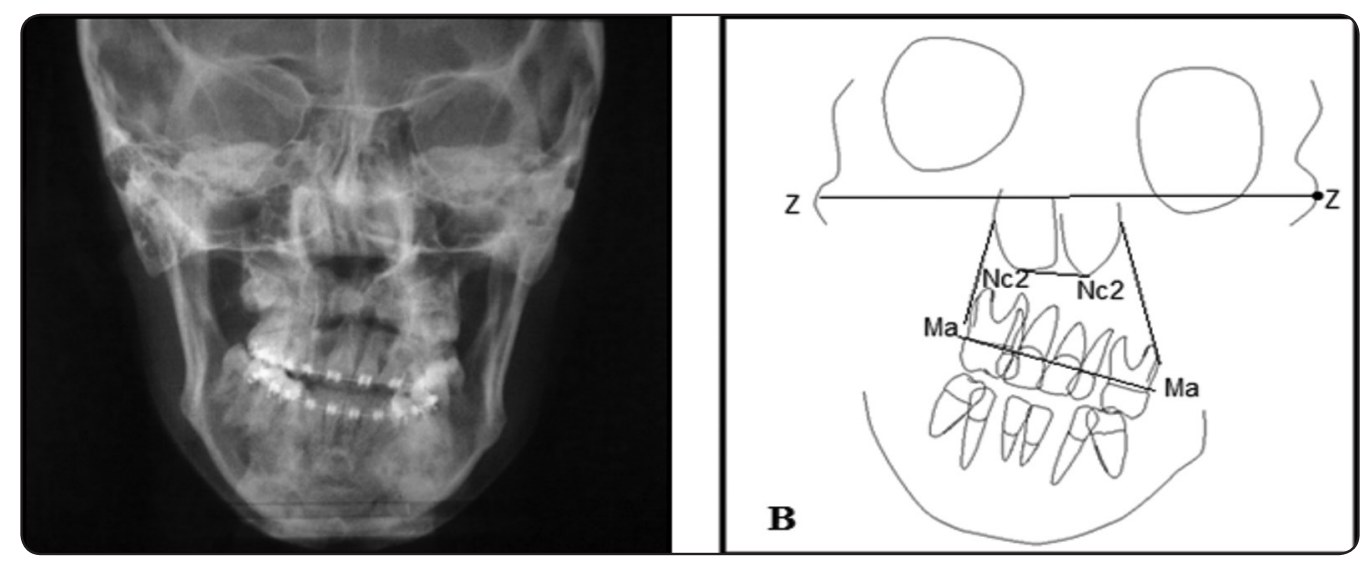

Fig. (1): A) Postero anterior cephalometric view showing collapsed maxilla of right side. B) Diagram showing tracing of postero anterior cephalometric view showing transverse deficiency of right maxilla. The width at the zygomatic process left and right was recorded $(\mathrm{Z}-\mathrm{Z})$ as a control measurement. The most inferior point of the piriform aperture was chosen (Nc2). Width at the nasal level was measured from $\mathrm{Nc} 2$ left to right $(\mathrm{Nc} 2-\mathrm{Nc} 2)$ for evaluation of the skeletal widening of the maxillary segments at the upper level. For measuring the skeletal widening of the maxilla at the most caudal level, point Ma was taken, situated at the intersection of the molar to the alveolar process left and right $(\mathrm{Ma}-\mathrm{Ma})$ and the segmental maxillary tipping due to the treatment at follow-up periods was evaluated by the change in the distance at the upper level (Nc2-Nc2) was subtracted from the change in distance at the caudal level (Ma-Ma) according to Berger et al. ${ }^{(15)}$. 
- Lateral cephalometric radiograph was taken to make tracing for each patient to detect the sagittal defect of maxilla and monitoring the progress of the patients postoperatively according to Silva et al. ${ }^{(12)}$.

- Computed tomography was utilized in axial, coronal and three dimensional (3D) cuts according to Swennen, et al. ${ }^{(13)}$, and Schwarz, et al. ${ }^{(14)}$.

\section{C) Study cast analysis}

- Study cast analysis was carried out for of the upper jaw cast to detect the inter canine, Interpremolar and Inter-molar distance according to Northway and Meade ${ }^{(16)}$ and Laudemann et al. (17).

- Palatal vault depth was recorded opposite to the second premolar and first molar preoperatively, three and six months postoperatively to monitor the progress of the vertical dimension of the patients according to Anttila et al. ${ }^{(9)}$.

- The upper and lower study models were articulated together to detect the amount of the cross bite, by measuring the distance from the midline to the cusp tip of the canine, second premolars and distobucal cusp of the first molars of the affected side and subtracted from the normal side according to Cross and McDonald $^{(18)}$.

\section{Distractor design}

The Transpalatal Distraction Osteogenesis (TPDO)* , (9, 18, 27 and 36 mm models, Martin, Germany) is a bone-borne distractor which is very easily placed and activated (Figure 2). The distraction device works on the principle of two counter rotating threads with identical pitches. By activating the distractor, the $2 \mathrm{~mm}$ long pins of the two abutment plates will penetrate the bone and the device is stabilized automatically. By inserting the two drill free screws, an additional fixation to the bone is necessary. After each activation/distraction, the distractor has to be blocked by securing the grey locking nut to avoid undesired movements.

\section{Surgical technique}

The size of distractor, position and the selection of the vector detected on the study cast according to Gerlach \& $\mathrm{Zahl}^{(19)}$ before distractor is sterilization. The surgical technique was performed in programmed steps for all patients as all patients were operated under general anesthesia. The patients were positioned with the head in an extended position.

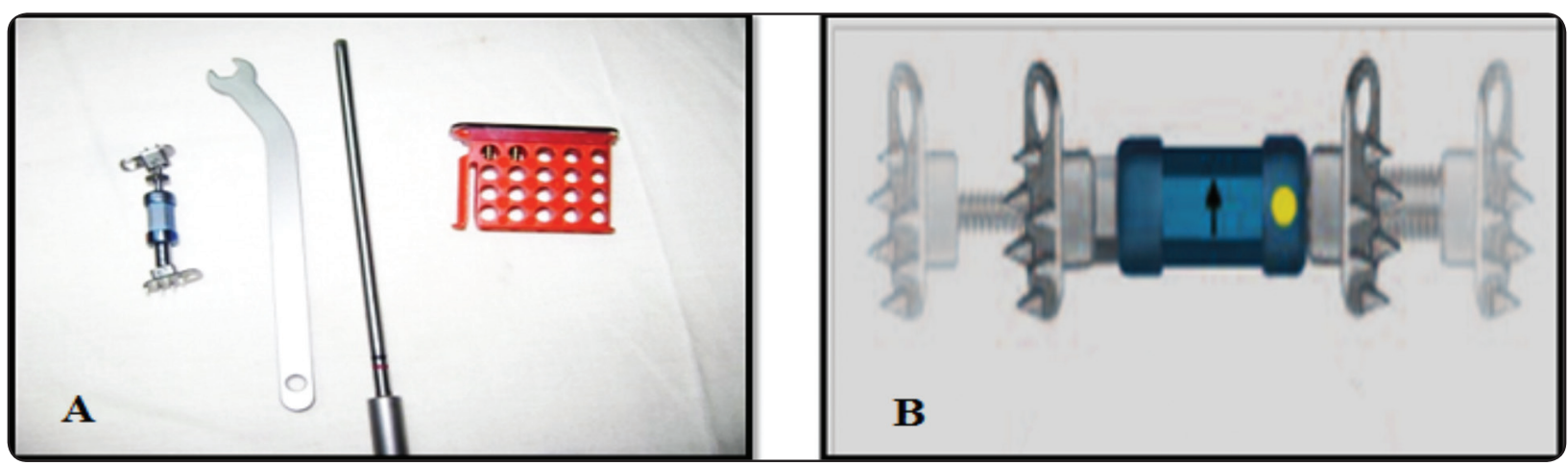

Figure (2): Martin distractor A) from right to left TPDO distractor, patient wrench, screwdriver and self-tapping screws its size (5 $\mathrm{mm}$ ). B) TBDO $2 \mathrm{~mm}$ long pins, color code and arrow for the direction of the activation.

KLS Martin GmbH + Co. KG 79224 Umkirch. Germany info@klsmartin.com. 
This was followed by scrubbing and draping of the surgical field. Intraoperatively; all patients received a standard dose of Unasyn ${ }^{\circledR} 1500 \mathrm{mg}^{*}$ and Decadron $^{\circledR} 4 \mathrm{mg}^{* *}$ I.V. Local anesthetic solution with a vasoconstrictor (Mepivacaine hydrochloride 2\% local anaesthesia with levonordefrin 1: 20000 vasoconstrictor) $^{* * * *}$ was infiltrated along the labial and palatal tissues and along the vestibular sulcus to aid in hemostasis and easier soft tissue dissection during the procedure.

The transpalatal distraction osteogenesis was temporarily fixed with the abutment plates on the mucosa over the roots of the first and second premolars to determine the distraction vector. The activation rod is in the midline and must not interfere with the lower teeth in occlusion. The distractor is slightly activated, thus the print of the plates is clearly visible in the mucosa. The distraction vector was oblique in relation to the occlusal plane, in order to correct the antero-posterior and the vertical deficiencies. Site of palatal incisions were marked and the distractor deactivated and removed according to Kuroe et al. ${ }^{(20)}$ (Figure 3).

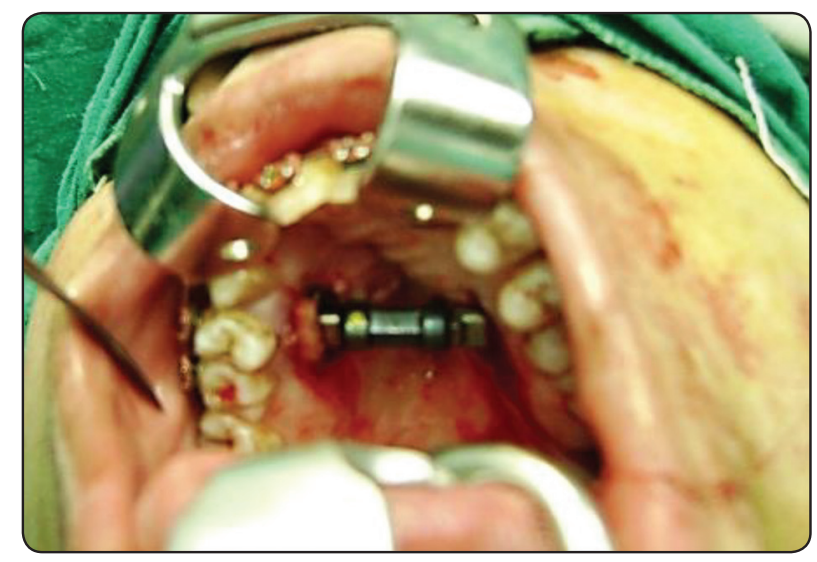

Fig. (3): Showing the site of palatal incision for fixation of the distractor.
A mucoperiosteal flap was elevated through long vestibular incision above the attached gingival by $3 \mathrm{~mm}$ starting from the mesial aspect of upper canine on the opposite side to the mesial aspect of the upper second molar on the affected side. The mucoperiosteum was carefully reflected exposing unilaterally the nasal floor and lateral aspect of the maxilla between the canine root and the infraorbital nerve. Posteriorly, the periosteum was undermined until the pterygomaxillary junction was identified. The nasal mucosa was dissected away from lateral nasal bone according to Kuroe et al. ${ }^{(20)}$ (Figure 4).

Osteotomy of the maxillary buccal bone was performed high, 3-5 $\mathrm{mm}$ away from the roots of the teeth and just below the zygoma, extended from the lateral maxillary wall to the pterygomaxillary fissure. It was important to perform adequate horizontal bone removal at the zygomatic buttress (width of the fissure bur with copious irrigation with normal saline to avoid bone necrosis at the osteotomy site) to allow lateral rotation of the osteotomized basal bone maxillary segment during transpalatal distraction. The maxillary segment was tested for movement to ensure that the distraction will be possible. In unilateral collapse, the osteotomy was stopped at the cleft edge if the alveolar cleft previously treated osteotomy extends in the alveolar graft as in (Figure 5).

The pterygomaxillary junction (posterior support) is released with a curved osteotome. The osteotomy extended through the palate (medianposterior support) with a fine curved osteotome placed in the vertical bony cut or in the alveolar cleft. The osteotome malleted through the hard palate toward the pterygomaxillary junction with a finger maintained on the palatal mucosa to guide the osteotome. Septal release is not performed. At this time, mobility of the osteotomized dentoalveolar

\footnotetext{
* Sulbactam/Ampicillin, Pfizer, Egypt, S.A.E., Cairo, A.R.E, under authority of Pfizer Inc. U.S.A.

** Decadron E.P.C.O.Co A.R.E.

*** Mepecaine-L, carpule, Alexandria Co. for pharmaceuticals and chemical Ind. Alexandria. Egypt.
} 


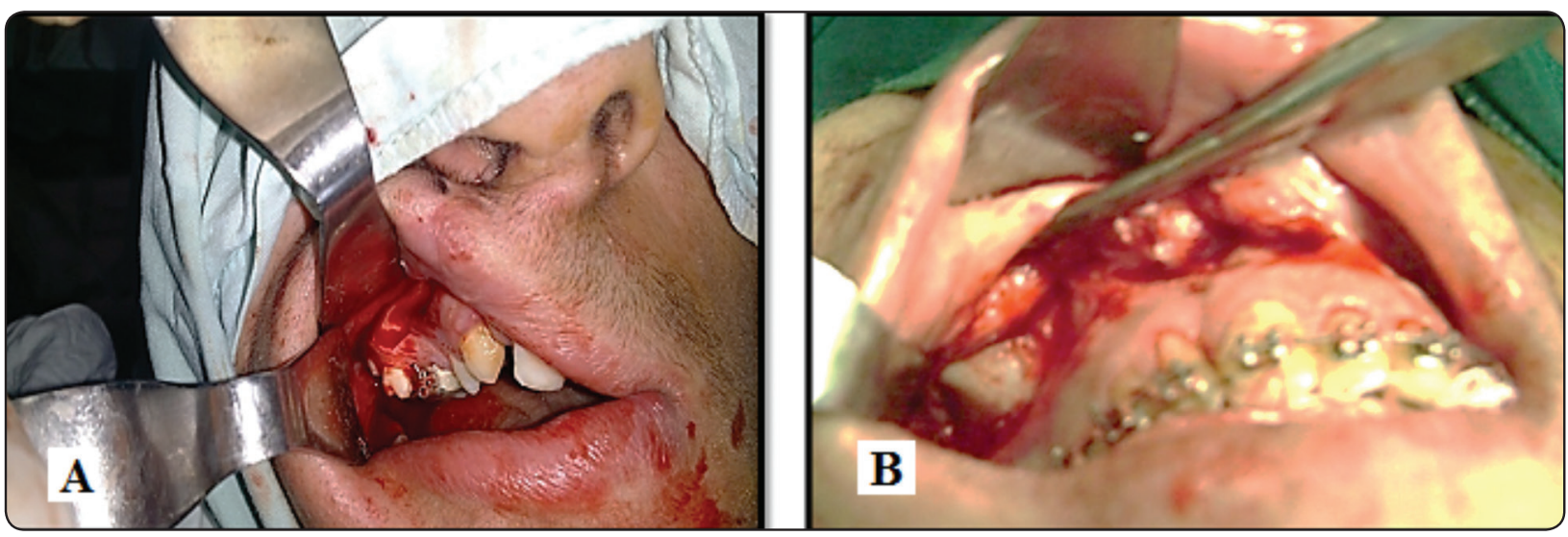

Fig. (4): Showing mucoperiosteal flap were elevated through long vestibular incision above the attached gingival by $3 \mathrm{~mm}$ for two groups. A) Group1: TransPalatal Distractor Osteogenesis of cleft palatal patient by TPDO. B) Group 2: TransPalatal expansion of cleft palatal patient by HYRAX.

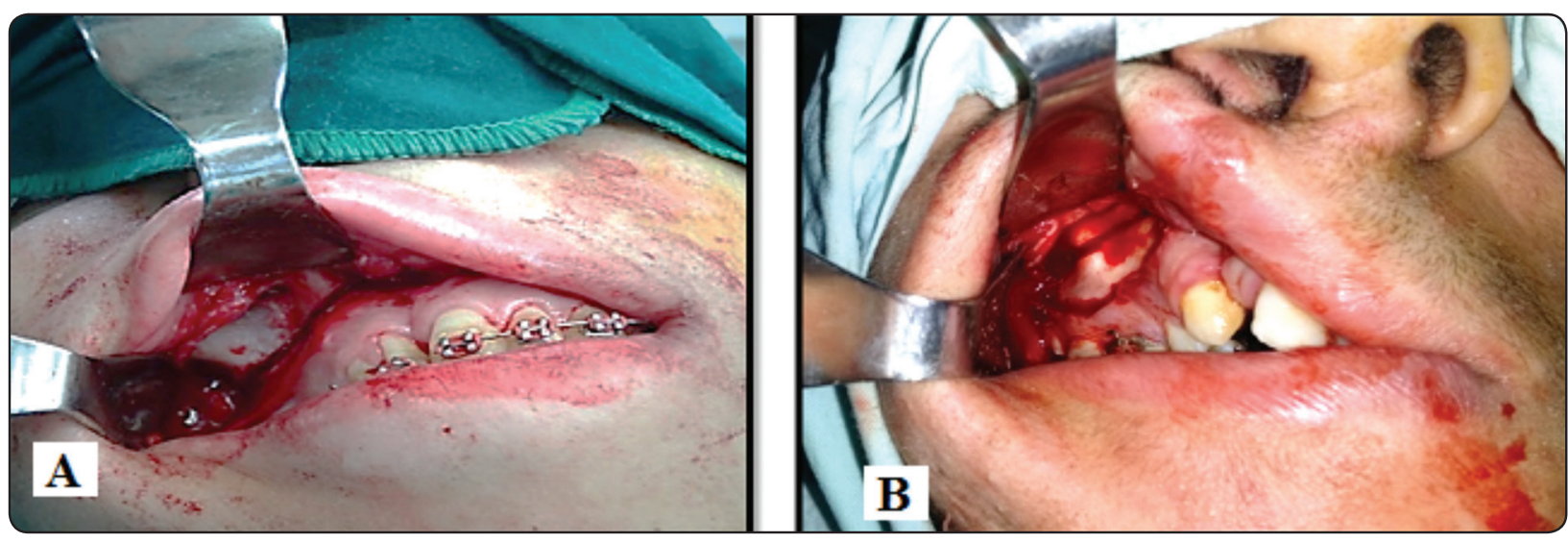

Fig. (5): Showing Osteotomy of the maxillary buccal bone was performed high, 3-5mm away from the roots of the teeth and just below the zygoma for two groups. A) Group1, B) Group 2

segment should be verified to ensure that all bony resistance is released. After infiltration with a vasoconstrictor (mepivicaine HCL 2\% with 1:20,000 levonordefrin) for local heamostasis, a horizontal incision was made on each vertical wall of the palatal vault corresponding to the canine premolars region in oblique position to allow three dimensional movements. Based on this incision, a small triangular mucoperiosteal flap was excised with the tip to the palatal midline.

The titanium abutment plates of the RPE distractor were horizontally fixed and the appropriate RPE distractor was fitted into the slots of the abutment plates. The distractor was then placed in the palate opposite premolar canine region then, once the osteotomized maxillary segment reach the desired position, the RPE distractor module was stabilized in the palatal bone (Figure 6). The oral mucosa on the buccal side was sutured over the defect closing the alveolar cleft if not repaired previously with soft tissue only Morris et al., ${ }^{(21)}$.The soft tissue incision was closed with absorbable suturing material (Vicryl 3/0).

\section{The postoperative care and recommendations for all patients}

Administration of broad spectrum antibiotics, non-steroidal anti-inflammatory drug for pain relief and an initial dose of corticosteroids immediately 


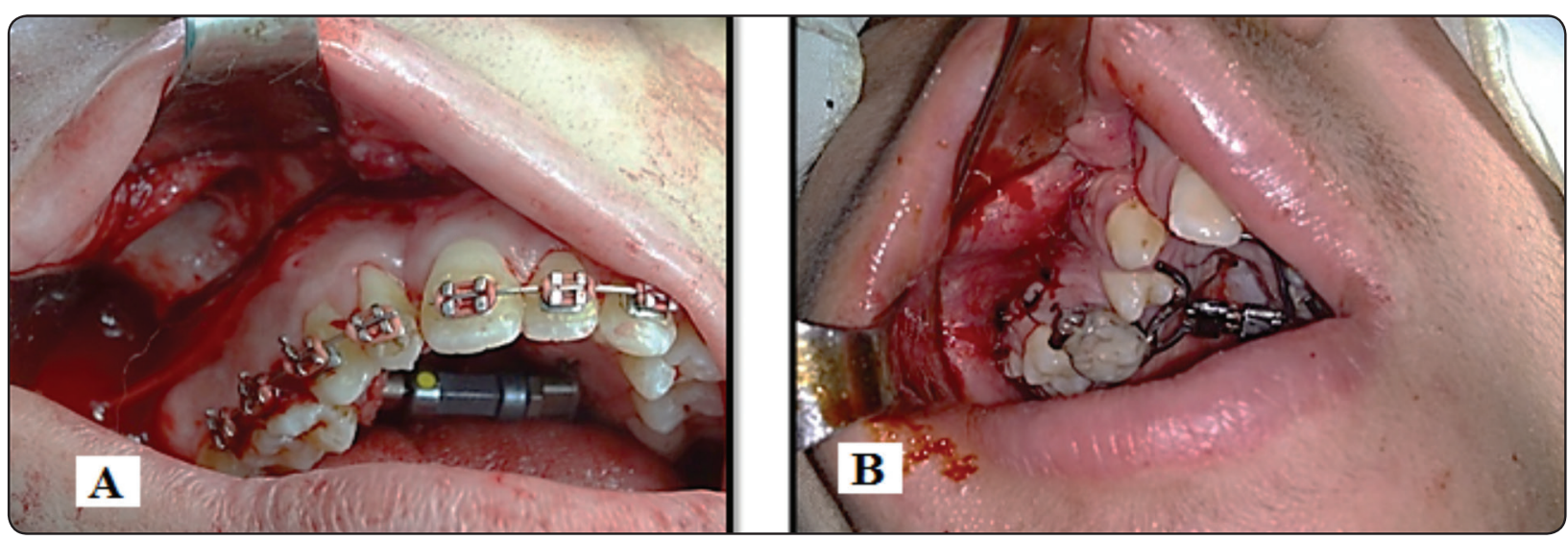

Fig. (6): Showing oral mucosa on the buccal side was sutured over the defect closing the alveolar cleft for two groups. A) Group1: TransPalatal Distractor Osteogenesis of cleft palatal patient by TPDO. B) Group 2: TransPalatal expansion of cleft palatal patient by HYRAX.

postoperative to reduce postoperative edema and multivitamins the patient was advised to avoid any physical activities or sports exercises that could cause an external shock, fall or similar impact. Extra-orally, elastic tapes covering the upper lip were applied as a pressure bandage to decrease postoperative edema. Post-operative instructions included using cold applications in the form of ice bags ten minutes every half an hour for the six hours post-operatively. The patients were instructed to avoid negative or positive pressure inside the nasal cavity such as oral and nasal blowing and instructed to maintain good oral hygiene with mouth wash and brushing the remaining teeth were explained to the patients and their families. Liquid diet was instructed for 2-3 days postoperatively; then a soft diet was instructed for one month, after which a normal diet was resumed.

\section{Latency period:}

The distractors were left 5-7 days (latency period) to allow soft callus formation.

\section{Activation period:}

After latency period, expansion was achieved at home with the patient key at a rate of $1 \mathrm{~mm}$ per day (one color code $=0.33 \mathrm{~mm}$ ) by rotating it downwards from cranially to caudally till the next color code appears and continue until the required transverse maxillary width was reached (three colors code $=$ one full turn $=$ one $\mathrm{mm}$ ) . The device was retained for 4 months for consolidation and removed under local anesthesia ${ }^{(8)}$.

\section{Postoperative evaluation}

Clinical evaluation included wound healing, infection signs at the operating site, bleeding and pain (which is detected by using visual analog scale (VAS) that was done by drawing a circle on the diagram to indicate the site of pain, and pain score from 0 to 10 where 0 is pain free and 10 is severe pain). Patient compliance and device looseness were also evaluated.

Intra oral photographic views (frontal open, frontal closed, posterior right and posterior left views) taken immediately post operatively then three months, six months and twelve months post operatively. Extra oral photographic views (frontal, lateral profile and 3/4 photographic views) were taken immediately post operatively then three months, six months and twelve months post operatively.

Radiological evaluation was done through posterior -anterior cephalometric view at three, six and twelve months postoperatively, lateral cephalometric view at three, six and twelve months 
postoperatively and axial, coronal and three dimensional (3D) CT scan at three, six and twelve months postoperatively.

Study cast analysis was carried out at three, six and twelve months postoperatively to detect inter canine, inter premolars and inter molars distances, palatal depth and palatal width.

\section{Data Management and Analysis:}

The data were recorded, tabulated and analyzed. The Statistics Package for Social Sciences (SPSS Inc., Chicago, IL, USA) was used for statistical analysis.

Statistical evaluation was performed by using Paired t-test for comparison the data within the studied groups and Student t-test for comparison the data between studied groups. Differences were considered significant when $\mathrm{P}$ value $<0.05$ and insignificant when $\mathrm{P}$ value $>0.05$.

\section{RESULTS}

\section{Clinical evaluation:}

- Wound healing at the sublabial, sulcular incision and palatal tissue

Within the first seven postoperative days uneventful primary wound healing was achieved in all patients on labial and buccal side and palatal tissue ulcers were observed around arms of transpalatal distractor which were healed spontaneously. The sutures were removed in the $15^{\text {th }}$ day postoperatively to avoid development of the oroantral fistula. No infection was noticed in the wound and overlying soft tissue.

\section{Patient compliance:}

The patients reported variable degrees of tolerable pain associated with activation of the distractor. No abnormal teeth mobility was seen during the latency period. Three patients showed accentuation of the palatal fistula resulted in more running fluids from the nose with drinking as a result of maxillary expansion. This problem was treated by closure of the fistula after removal of the transpalatal distractor.

Moderate to severe soft tissue edema of upper lip and cheek was apparent at the first four days of surgery. Then it was gradually resolved by antiedematous and warm applications extraorally at the site of surgery. Patients didn't exhibit any difficulty in maintaining adequate nutrition throughout duration of the study.

\section{- Device looseness}

There was device loosening in case no. 8 refixed to its position under local anesthesia by using emergency screws.

\section{- Photographic assessment}

Intra-oral photographs of the upper jaw were taken for the patients preoperatively and postoperatively to compare the post distraction outcome of the upper jaw with the pre-distracted (collapsed) upper jaw for the patients (Figure 7).

\section{- Radiographic evaluation}

- Lateral cephalometric view analysis

After tracing for each patient lateral cephalometric view the postoperative antero-posterior changes according to $\mathrm{SNA}^{0}$ was recorded and assessed; the results are summarized in table (1). There was increase in $\mathrm{SNA}^{0}$ postoperatively which indicated an increase in sagittal dimension postoperatively which was found to be statistically significant.

TABLE (1): Statistical analysis of SNA degree on lateral cephalometric view

\begin{tabular}{|l|c|c|}
\hline & TPDO Mean & HYREX Mean \\
\hline Preoperative & $\mathbf{7 4 . 0 9 2}$ & $\mathbf{7 4 . 0 9 2}$ \\
\hline After 3 month & 79.000 & 76.200 \\
\hline After 6 month & $\mathbf{7 8 . 5 4 2}$ & 75.192 \\
\hline After 12 month & $\mathbf{7 8 . 5 4 2}$ & 75.192 \\
\hline Relapse & $\mathbf{0 . 4 5 8}$ & 1.01 \\
\hline
\end{tabular}

* P<0.05 (significant) 

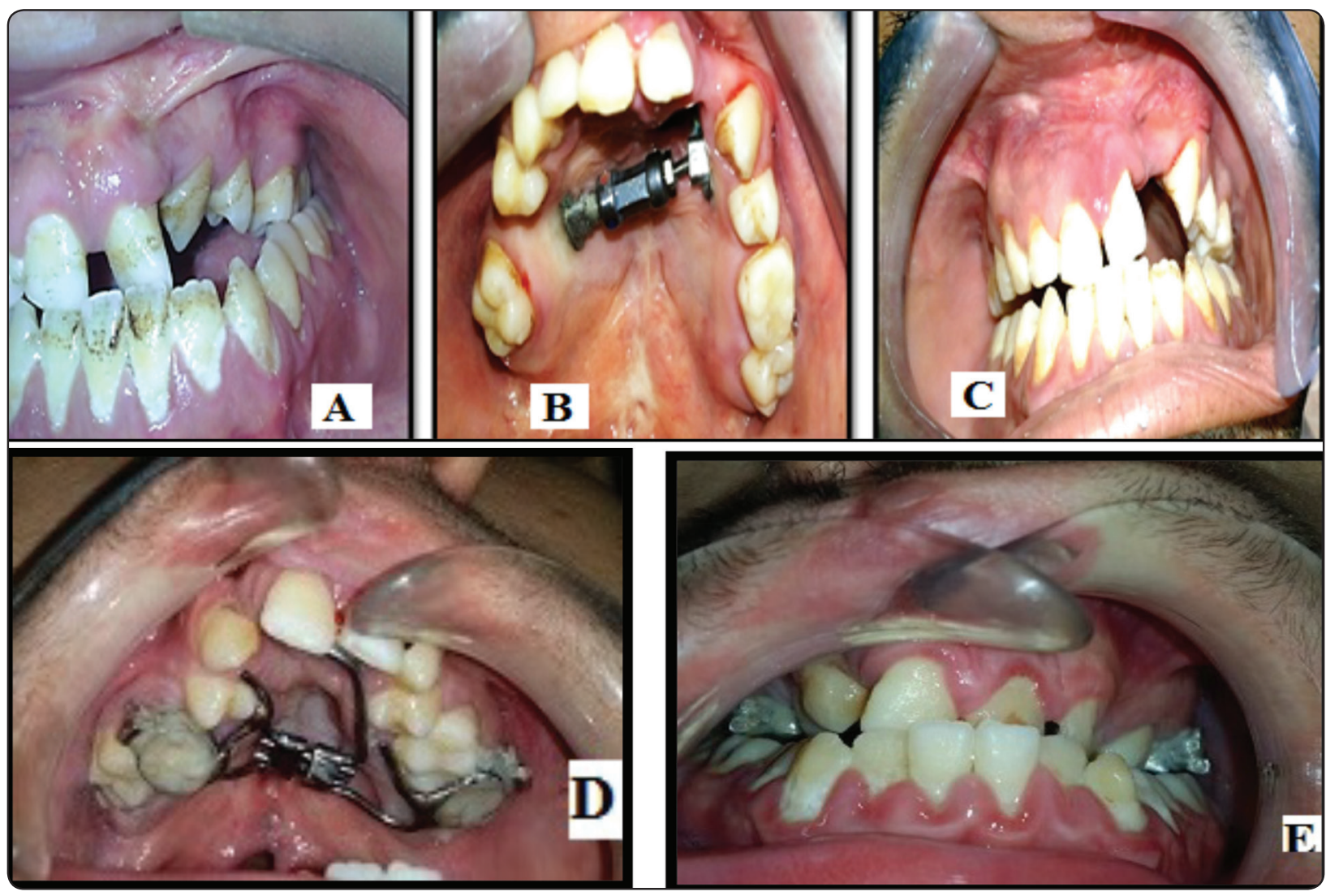

Fig. (7): A) Preoperative photograph showing left cross bite and collapsed maxilla, B) Three months post-distraction showing correction of collapsed maxilla. C) Six months postoperative photograph showing corrections of the cross bite which is amenable for orthodontic correction of teeth on the affected side, D\&E) showing correction of collapsed maxilla using HYRAX appliance.

- Posteroanterior cephalometric view analysis for evaluating the skeletal widening of the maxillary segments at the upper level.

The most inferior point of the piriform aperture was chosen (Nc2). Width at the nasal level was measured from $\mathrm{Nc} 2$ left to right $(\mathrm{Nc} 2-\mathrm{Nc} 2)$ $\mathrm{mm}$. The results (summarized in table 2) showed an increase of ( $\mathrm{Nc} 2-\mathrm{Nc} 2)$ postoperatively indicating an increase of upper level of maxilla and width of the nose which was found to be statistically significant.

- Posteroanterior cephalometric view analysis for evaluating the skeletal widening of the maxilla at the most caudal level.
Point (Ma) was taken, situated at the intersection of the molar to the alveolar process left and right $(\mathrm{Ma}-\mathrm{Ma})$. There was increase of $(\mathrm{Ma}-$ Ma) postoperatively indicating an increase of caudal level of maxilla which was statistically significant

- Postero-anterior cephalometric view analysis for evaluating the amount of segmental maxillary tipping

The change in distance at the upper level (Nc2$\mathrm{Nc} 2$ ) was subtracted from the change in distance at the caudal level $(\mathrm{Ma}-\mathrm{Ma})$; where the changes were statistically insignificant. (Figure 8) 

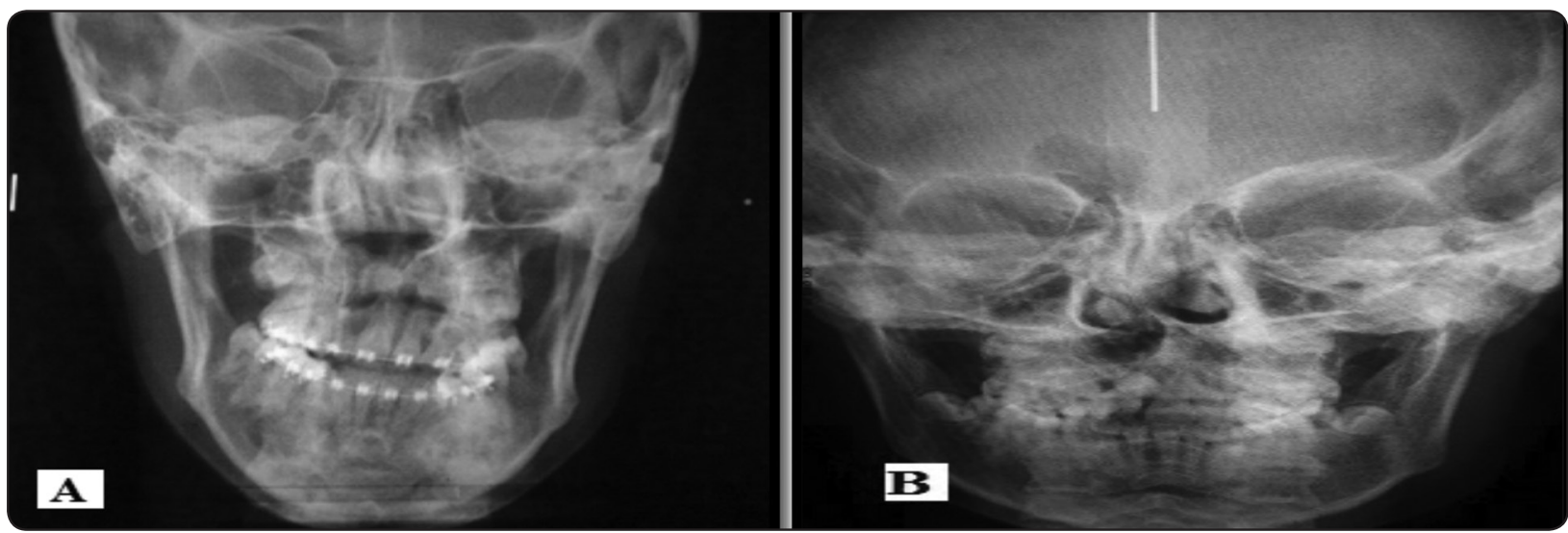

Fig. (8): A) Preoperative PA cephalometric view showing collapsed maxilla of right side. B) Postoperative PA view showing an increase of upper level of maxilla and width of the nose.

TABLE (2): Statistical analysis of caudal and cephalic widening of mouth

\begin{tabular}{ccccccc}
\hline & \multicolumn{2}{c}{ (Nc2-Nc2 mm) PA ceph } & \multicolumn{2}{c}{ (Ma-Ma mm) PA ceph } & \multicolumn{2}{c}{ (Ma-Ma mm) PA ceph. Mxillary } \\
& TPDO & HYREX & TPDO & HYREX & TPDO & HYREX \\
\hline Preoperative & 18.042 & 18.042 & 57.000 & 56.040 & 0 & 0 \\
3 month & 22.000 & 22.000 & 61.375 & 62.243 & 0.333 & 2.55 \\
6 month & 21.500 & 20.500 & 60.917 & 59.247 & 0.333 & 1.5 \\
12 month & 21.417 & 20.401 & 60.875 & 59.247 & 0.333 & 1.5 \\
Relapse & 0.583 & 1.599 & 0.5 & 3.004 & -- & -- \\
\hline
\end{tabular}

* $\mathrm{P}<0.05$ (significant)

\section{Computed Tomography measurement of linear measurement at canine}

The mean of distance measured from fixed points for each patient on CT at buccal bone of upper canine to the corresponding point on opposite side. There was increase in linear measurement at the canine on C.T. scan and it was statistically significant (table $\mathbf{3}$ ).

○ Computed Tomography measurement of Linear measurement at first molar

The mean of distance measured from fixed points for each patient on CT at buccal bone of upper first molar to the corresponding point on opposite side. There was a statistically significant increase in mean measurements.
Dental cast measurements (table 4)

\section{- The intercanine distance}

Through the tip of the cusp of the canine of the cleft side to the tip of the cusp of the canine in another side. There was a statistically significant increase in mean measurements.

\section{- The interpremolars distance}

This was measured from the tip of the buccal cusp of the second premolar in the cleft side to the tip of the buccal cusp of the second premolar of the normal side. There was a statistically significant increase in mean measurements. 


\section{- The intermolars distance}

This was measured from the tip of the distobuccal cusp of the first molar of the cleft side to the distobuccal cusp of the first molar of the normal side. There was a statistically significant increase in mean measurements.

- Palatal depth:

This was evaluated at premolars region by measuring the palatal vault depth opposite to the second premolar. There was a statistically significant increase in mean measurements (Figure 9).

\section{○ Palatal width:}

This was measured at $5 \mathrm{~mm}$ occlusal to the maximal palatal depth. There was a statistically significant increase in mean measurements (table 5).

TABLE (3): Statistical analysis of measurement of linear measurement at canine and molar on CT.

\begin{tabular}{ccccc}
\hline & \multicolumn{2}{c}{ Canine } & \multicolumn{2}{c}{ Molars } \\
& TPDO & HYREX & TPDO & HYREX \\
\hline Preoperative & 20.081 & 21.121 & 52.416 & 53.321 \\
After 3 month & 31.833 & 32.733 & 57.750 & 58.750 \\
After 6 month & 31.251 & 29.563 & 57.710 & 55.950 \\
After 12 month & 31.215 & 29.528 & 57.700 & 55.910 \\
\hline
\end{tabular}

$* P<0.05$ (significant)

TABLE (4): Statistical analysis of measurement of intercanine premolar and molar distance

\begin{tabular}{|l|c|c|c|c|c|c|}
\hline & \multicolumn{2}{|c|}{ intercanine distance $(\mathrm{mm})$} & interpremolars distance $(\mathrm{mm})$ & \multicolumn{2}{c|}{ intermolars distance $(\mathrm{mm})$} \\
\cline { 2 - 7 } & TPDO & HYRAX & TPDO & HYRAX & TPDO & HYRAX \\
\hline Preoperative & 18.167 & 19.167 & 39.417 & 40.347 & 51.500 & 52.500 \\
\hline After 3 month & 30.583 & 30.566 & 45.917 & 47.247 & 57.250 & 59.250 \\
\hline After 6 month & 29.917 & 28.219 & 45.417 & 44.626 & 57.000 & 56.000 \\
\hline After 12 month & 29.875 & 28.215 & 45.375 & 44.439 & 56.958 & 55.900 \\
\hline
\end{tabular}

$* P<0.05$ (significant)

TABLE (5): Statistical analysis palatal depth at premolars.

\begin{tabular}{|c|c|c|c|c|c|}
\hline & \multicolumn{3}{|c|}{ Palatal depth } & \multicolumn{2}{|c|}{ Palatal width } \\
\hline & PTDO & HYRAX & Mean & PTDO Mean & HYRAX Mean \\
\hline Preoperative & 17.042 & \multicolumn{2}{|c|}{17.042} & 17.833 & 18.343 \\
\hline After 3 month & 19.167 & \multicolumn{2}{|c|}{16.167} & 21.167 & 20.016 \\
\hline After 6 month & 18.850 & \multicolumn{2}{|c|}{16.167} & 20.958 & 19.658 \\
\hline After 12 month & 18.842 & \multicolumn{2}{|c|}{16.257} & 20.083 & 19.137 \\
\hline
\end{tabular}

$* P<0.05$ (significant) 


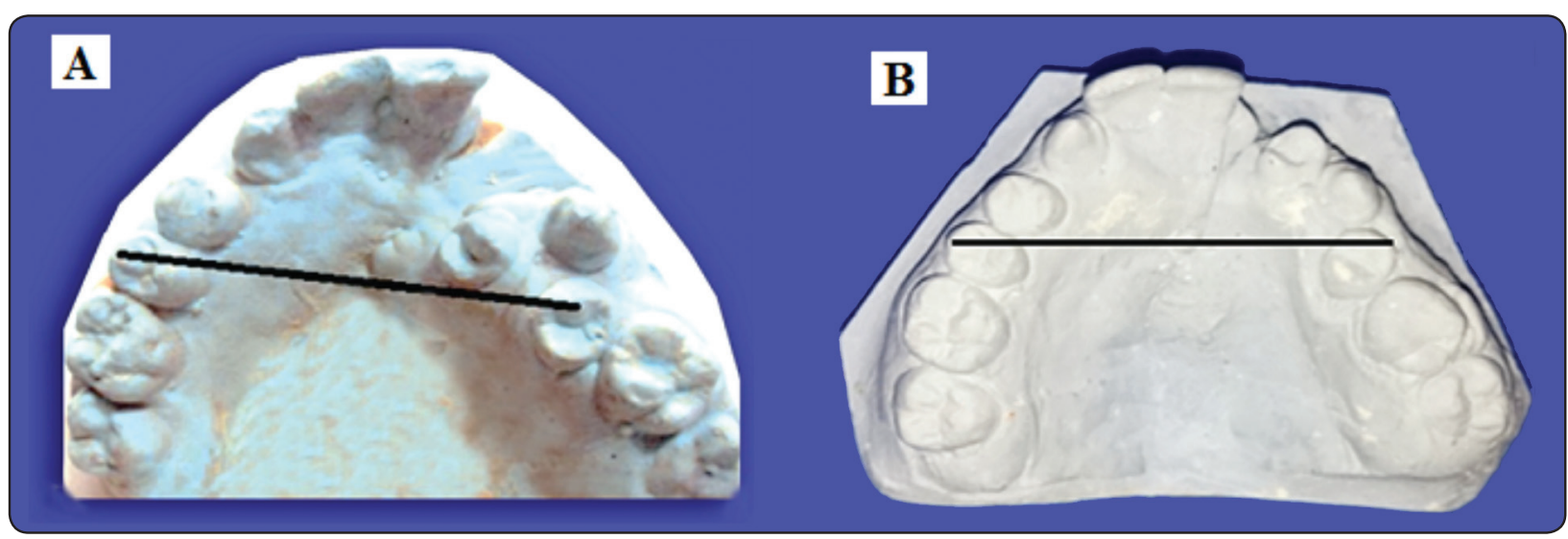

Fig. (9): A) Preoperative the interpremolars distance was $(40 \mathrm{~mm})$. B) Twelve month postoperatively the interpremolar distance was $(46 \mathrm{~mm})$.

\section{DISCUSSION}

Maxillofacial growth disturbances in UCLP patients can present with an asymmetric transverse maxillary deficiency caused by collapse of the lateral segment at the cleft side with sagittal and vertical dentofacial deformity. Larger transverse deficiency at the canine bicuspid region compared with the molar region ${ }^{(7)}$.

This study included Sixteen patients with unilateral collapsed maxilla after repair of the cleft lip and palate for the comparison of the results obtained by the normal side (eight patients group1 (TPDO) ; (3) males and (5) females and eight patients group2 (HYRAX) ; (2) males and (6) females patients with unilateral cleft lip and palate) the age of the patients above 14 years because at this age the sutural growth of the maxilla stopped and the traditional orthodontic treatment cannot give a result this is in accordance with Pintoet al. ${ }^{(22)}$.

Le Forte- I osteotomy in this study was extended to pterygoid plate disjunction to sure the bodily and free movement of the affected segment without torque, this in accordance with Silverstein and Quinn ${ }^{(23)}$. The use of group1 (TPDO) was superior to all other types of palatal distractors as it have $2 \mathrm{~mm}$ long pins of the two abutment plates will penetrate the bone and the device is then stabilized automatically and only two drill free screws for additional fixation to the bone. Decreasing the number, length $(5 \mathrm{~mm})$ and type of screws used (drill free screws) will further decrease the chance of roots damage and allow good blood supply.

The selected distractor vector (TPDO) was in oblique direction anteroposteriorly which give opportunity for correction of the collapsed side in both transverse and sagittal dimensions moreover, its fixation at two levels allowed the correction of the vertical dimension. The distraction osteogenesis allows new bone and soft tissue formation as well as stretching the scar tissue which is an important cause of relapse in cleft palate patients making the distraction superior to all other techniques used for treatment of these cases. Furthermore, in cleft patients, because the appliance is rigidly fixed to the osteotomized bone in bone-borne device, undesired movement of the bony segments is eliminated. Another important point is the ossification of the bone osteotomies which begins during the distraction period and continues during the retention time, where, the transpalatal distraction offers a high retention stability without or with a minimal relapses as reported by Treutlein et al. ${ }^{(24)}$ and this result was parallel to the result of this study where the relapses was in between $0.5-1 \mathrm{~mm}$. 
The upper level of the skeletal maxillary segments became more wider than the preoperatively where it was preoperatively (mean) $18.042 \mathrm{~mm}$ then after three months postoperatively became $22 \mathrm{~mm}$ but at six to twelve months had slight relapse $(0.5-0.6 \mathrm{~mm})$. The caudal level of the skeletal maxillary segments increased than the preoperatively where it was preoperatively (mean) 57 mmthen after three months postoperatively became 61.375 mmwhile at six to twelve months relapse happened with $0.5-0.6 \mathrm{mmaccording}$ to postero-anteriorcephalometric view and this results in agreement with the results obtained by Chung and Goldman ${ }^{(25)}$ and also in accordance with Chung and Font ${ }^{(4)}$. Skeletal and dental changes in the sagittal, vertical, and transverse dimensions after rapid palatal expansion where (Nc2-Nc2) preoperatively was (mean) 17.8 mmand postoperatively became20.4 mmwith relapse of $0.8 \mathrm{~mm}$ at twelve months and (Ma-Ma) was preoperatively (mean) $58.8 \mathrm{~mm}$ then became postoperatively $62.1 \mathrm{mmwith}$ a relapse of 0.7 mmat twelve months.

In this study the relapse at the upper level was 2.5 - 3\%while at the caudal was $0.86-0.96 \%$ and this was in agreement with a study achieved by Koudstaal et al.(7) where they determined that the relapse at the upper level was $4.7 \%$ and relapse at the caudal level however was $0.9 \%$.

The results of the segmental maxillary tipping measurements on the PA cephalograms was found 0.7 (SD of 2.6) in the bone-borne device but this was not significant in studies of Anttila, et al (9), Doruk et $\mathrm{al}^{(10)}$ and Christie et al. ${ }^{(26)}$ and this was similar to the results found in our study.

There was increase in mean of linear measurements at the first molar according to coronal C.T. scan where it was (mean) preoperatively $52.416 \mathrm{~mm}$ and became $57.71 \mathrm{~mm}$ three months post operatively and at six and twelve months postoperatively became 57.7 mmand these results were similar to results obtained by Elmohandas and Foda ${ }^{(27)}$ determined the effect of bone borne transpalatal distractor on collapsed maxilla.

In this study there was increase in mean measurements interpremolars distance according to dental cast where it was (mean) preoperatively $39.414 \mathrm{~mm}$ and became $45.917 \mathrm{~mm}$ three months post operatively, at six months postoperatively (mean) $45.417 \mathrm{~mm}$ and at twelve months postoperatively $45.375 \mathrm{~mm}$ this result similar to results obtained by Berger, et al ${ }^{(15)}$ where they measured that mean preoperative $37.2 \mathrm{~mm}$ and at the end of follow up period was $44.3 \mathrm{~mm}$. The relapse at premolar level was $0.1-0.3 \mathrm{~mm}$. according to Koudstaal, et al (7), this with agreement of the results of our study where the relapse was $0.5-0.542 \mathrm{~mm}$.

In this study there was increase in palatal depth at the premolars according to dental cast records where it was preoperatively (mean) $17.042 \mathrm{~mm}$ and became (mean) $19.167 \mathrm{~mm}$ at three months postoperatively the percentage of increase was $12.469 \%$ while at six and twelve months postoperatively became (mean) $18.850-18.842 \mathrm{~mm}$ with a percent of change $10.562 \%$ this results better than results obtained by Chung, et al (28) , Chung and Font (4), Doruk, et al ${ }^{(10)}$ and Koudstaal, et al ${ }^{(7)}$ where there was a decrease in palatal depth at the premolars where it was preoperatively $20.3 \mathrm{~mm}$ and postoperatively $20.2 \mathrm{~mm}$.

In this study the maxilla moved slightly downward. The downward movement of the maxilla explained by the direction of the lateral corticotomy. This osteotomy line is generally slanting slightly downward from the nasal aperture to the zygomatic buttress, and the position of the RPE distractor in oblique line and fixed in the affected side at lower level than in normal side this position permits downward movement of the collapsed side with increase of the palatal depth and vertical dimension of the maxilla while in Chung, et al (28) , Chung and Font ${ }^{(4)}$, Doruk, et al ${ }^{(10)}$ and Koudstaal, et al ${ }^{(7)}$ the position of the palatal distractor was transverse and parallel this position increase palatal width and decrease palatal depth. 


\section{CONCLUSION}

The results of this study shows significant difference between the pre and post distraction proving that Transpalatal distraction osteogenesis (GROUP1) of collapsed maxilla in cleft palate patients give a good results without significant relapse due to complete freeing of the affected side by Le-Forte I and pterygoid plate disjunction and adequate consolidation period. Relapse in group2 (HYRAX) is more than group1 (TPDO). Segmental maxillary tipping does not affect relapse in TPDO. The oblique position of the TPDO allowed the three dimensional correction of the affected maxilla. The canine- premolar region mostly improved due to the position of the TPDO. Reliable evaluation was obtained by using lateral, postero-anterior cephalometric views and study cast.

\section{REFERENCES}

1. Wiltfang J, Hirschfelder U, Neukam FW, Kessler P. Longterm results of distraction osteogenesis of the maxilla and midface. British journal of oral \& maxillofacial surgery. 2002 Dec;40(6):473-9.

2. Riediger D, Poukens JM. Le Fort III osteotomy: a new internal positioned distractor. Journal of oral and maxillofacial surgery.2003 Aug;61(8):882-9.

3. Meyer U, Kleinheinz J, Joos U. Biomechanical and clinical implications of distraction osteogenesis in craniofacial surgery. Journal of cranio-maxillo-facial surgery.2004 Jun;32(3):140-9.

4. Chung $\mathrm{CH}$, Font B. Skeletal and dental changes in the sagittal, vertical, and transverse dimensions after rapid palatal expansion. American journal of orthodontics and dentofacial orthopedics. 2004 Nov;126(5):569-75.

5. Mommaerts MY. Transpalatal distraction as a method of maxillary expansion.British journal of oral \& maxillofacial surgery. 1999 Aug;37(4):268-72.

6. Günbay T, Akay MC, Günbay S, Aras A, Koyuncu BÖ, Sezer B. Transpalatal Distraction Using Bone-Borne Distractor: Clinical Observations and Dental and Skeletal Changes. Journal of Oral and Maxillofacial Surgery. 2008; 66(12):2503-14.
7. Koudstaal MJ, van der Wal KG, Wolvius EB, Schulten AJ. The Rotterdam Palatal Distractor: introduction of the new bone-borne device and report of the pilot study. International journal of oral and maxillofacial surgery. 2006 Jan;35(1):31-5.

8. Vyas RM, Jarrahy R, Sisodia M, Jourabchi N, Wasson KL, Bradley JP. Bone-borne palatal distraction to correct the constricted cleft maxilla. Journal of craniofacial surgery. 2009 May;20(3):733-6.

9. Anttila A, Finne K, Keski-Nisula K, Somppi M, Panula K, Peltomaki T. Feasibility and long-term stability of surgically assisted rapid maxillary expansion with lateral osteotomy. European journal of orthodontics. 2004 Aug;26(4):391-5.

10. Doruk C, Bicakci AA, Basciftci FA, Agar U, Babacan H. A comparison of the effects of rapid maxillary expansion and fan-type rapid maxillary expansion on dentofacial structures. The Angle orthodontist. 2004 Apr;74(2):184-94.

11. Koudstaal MJ, Poort LJ, van der Wal KG, Wolvius EB, Prahl-Andersen B, Schulten AJ. Surgically assisted rapid maxillary expansion (SARME): a review of the literature. International journal of oral and maxillofacial surgery. 2005 Oct;34(7):709-14.

12. da Silva Filho OG, Boas MC, Capelozza Filho L. Rapid maxillary expansion in the primary and mixed dentitions: a cephalometric evaluation.American journal of orthodontics and dentofacial orthopedics. 1991 Aug;100(2):171-9.

13. Swennen GR, Mollemans W, Schutyser F. Threedimensional treatment planning of orthognathic surgery in the era of virtual imaging. Journal of oral and maxillofacial surgery . 2009 Oct; 67(10):2080-92.

14. Schwarz GM, Thrash WJ, Byrd DL, Jacobs JD. Tomographic assessment of nasal septal changes following surgical-orthodontic rapid maxillary expansion. American journal of orthodontics. 1985 Jan;87(1):39-45.

15. Berger JL, Pangrazio-Kulbersh V, Borgula T, Kaczynski R. Stability of orthopedic and surgically assisted rapid palatal expansion over time. American journal of orthodontics and dentofacial orthopedics .1998 Dec;114(6):638-45.

16. Northway WM, Meade JB, Jr. Surgically assisted rapid maxillary expansion: a comparison of technique, response, and stability. The Angle orthodontist. 1997;67(4):309-20.

17. Laudemann K, Petruchin O, Nafzger M, Ballon A, Kopp S, Sader RA, et al. Long-term 3D cast model study: 
bone-borne vs. tooth-borne surgically assisted rapid maxillary expansion due to secondary variables. Oral and maxillofacial surgery. 2010 Jun;14(2):105-14.

18. Cross DL, McDonald JP. Effect of rapid maxillary expansion on skeletal, dental, and nasal structures: a postero-anterior cephalometric study. European journal of orthodontics. 2000 Oct;22(5):519-28.

19. Gerlach KL, Zahl C. Transversal palatal expansion using a palatal distractor. Journal of orofacial orthopedics .2003 Nov;64(6):443-9.

20. Kuroe K, Iino S, Shomura K, Okubo A, Sugihara K, Ito G. Unilateral advancement of the maxillary minor segment by distraction osteogenesis in patients with repaired unilateral cleft lip and palate: report of two cases. The Cleft palatecraniofacial journal. 2003 May;40(3):317-24.

21. Morris DO, Roberts-Harry D, Mars M. Dental arch relationships in Yorkshire children with unilateral cleft lip and palate. Cleft palate-craniofacial journal. 2000 Sep;37(5):453-62.

22. Pinto PX, Mommaerts MY, Wreakes G, Jacobs WV. Immediate postexpansion changes following the use of the transpalatal distractor. Journal of oral and maxillofacial. 2001 Sep;59(9):994-1000.

23. Silverstein K, Quinn PD. Surgically-assisted rapid palatal expansion for management of transverse maxillary deficiency. Journal of oral and maxillofacial surgery. 1997 Jul;55(7):725-7.

24. Treutlein C SGaBJ, et al. Transpalatinale distraktioneine alternative methode der transversalen expansion des oberkiefers. Dtsch Zahnarztlz. 2002:57S: 19.

25. Chung $\mathrm{CH}$, Goldman AM. Dental tipping and rotation immediately after surgically assisted rapid palatal expansion. European journal of orthodontics. 2003 Aug;25(4):353-8.

26. Christie KF, Boucher N, Chung CH. Effects of bonded rapid palatal expansion on the transverse dimensions of the maxilla: a cone-beam computed tomography study. American journal of orthodontics and dentofacial orthopedics. 2010 Apr;137(4 Suppl):S79-85

27. Elmohandas and Foda. Effect of Bone Borne Transpalatal Distractor on Both Collapsed Maxilla and Maxillary AirSinus. Cairo Dental Journal 2009;25 September (3):387:95.

28. Chung CH, Woo A, Zagarinsky J, Vanarsdall RL, Fonseca RJ. Maxillary sagittal and vertical displacement induced by surgically assisted rapid palatal expansion. American journal of orthodontics and dentofacial orthopedics . 2001 Aug;120(2):144-8. 